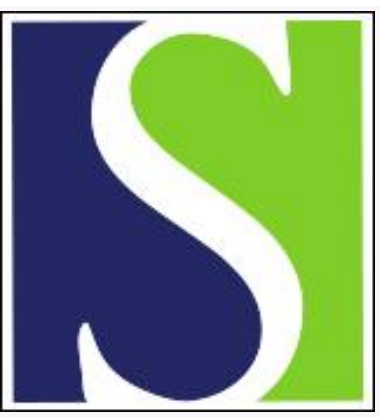

Scand J Work Environ Health 2010;36(2):81-84

https://doi.org/10.5271/sjweh.2902

Published online: 03 Feb 2010, Issue date: 00 Mar 2010

\title{
Shift work and health - how to proceed?
}

by Härmä $M$, Kecklund $G$

Affiliation: Finnish Institute of Occupational Health, Fl-00250 Helsinki, Finland. mikko.harma@ttl.fi

Refers to the following texts of the Journal: 2010;36(2):96-108 2010;36(2):163-179 2010;36(2):150-162 2010;36(2):85-95 2010;36(2):109-121 2010;36(2):121-133 2009;35(3):163-179 2008;34(1):5-22 2008;34(1):1-3 2006;32(6):502-514 2009;35(3):193-202 2008;34(3):198-205 2009;35(3):180-187 2005;31(5):325-327 2010;36(2):134-141 2010;36(2):142-149 2009;35(5):361-367 1989;15(3):165-179 1999;25(2):85-99 2004;30(1):1-3

The following articles refer to this text: 2010;36(6):515-516; 2012;38(4):291-297; 2015;41(3):268-279; 2015;41(4):413-416; 2018;44(1):80-87; 2020;46(1):1-4

Key terms: editorial; health; shift work

This article in PubMed: www.ncbi.nlm.nih.gov/pubmed/20126970

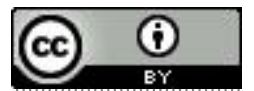




\section{Shift work and health - how to proceed?}

In Europe today, only a quarter of the employed workforce are engaged in regular day work. The rest of the employees and over $90 \%$ of self-employed workers have irregular or flexible working hours; they work in shifts $(22 \%$ of men and $11 \%$ of women) or more or less odd hours $(1,2)$. The established morningto-afternoon regular office-time is, therefore, becoming more of a rarity.

This special issue of the Scandinavian Journal of Work, Environment \& Health includes a number of reviews and discussion papers on shift work and health, focusing on cardiovascular disease (3), cancer (4), eating habits (5); and gastrointestinal disorders (6). Two additional papers review the current knowledge on countermeasures to the negative effects of shift work, covering both individual coping strategies for managing night work (7) and ergonomic shift scheduling to reduce sleep-wake disturbances (8). Adding here the two recently published systematic reviews on shift work and ischemic heart disease (9) and shift work and cancer (10), we could say that before you lies the core evidence on the effects of shift work on health.

But what is the extent of the evidence? Is the issue sufficiently covered in order to utilize the knowledge in practice? Which countermeasures should we select and how should we proceed?

Shift work has been a health issue in Europe since the end of the $18^{\text {th }}$ century (11), which explains why a large amount of studies on shift work and health have been carried out during the years. The effects of shift work on sleep and alertness are well established, the strongest evidence stemming from field and intervention studies (eg, 8, 12-15) showing that changes in shifts influence sleep, alertness, and fatigue. Since sleep and performance are closely related, shift work and long working hours are also a safety risk (16). Two recent systematic reviews $(9,10)$ suggest that shift work probably increases the risk for ischemic heart disease and breast cancer. The "probably" means that, although the evidence for causal associations is still limited, the observed associations are unlikely to be explained by chance, bias, or confounding. In this issue, a population-based case-control study by Pesch et al (17) adds to the existing evidence, showing that long-term night work is associated with a modestly [odds ratio (OR) 2.48], but not significantly increased risk of breast cancer (17). Moreover, in a 14-year prospective study, Suwazono et al (18) confirmed that very long follow-up times are needed in order to detect the effects of shift work on health - the threshold number of years in shift work that caused a $5 \%$ increase in blood cholesterol was found to be $\geq 21$ years among middle-aged Japanese workers. In this issue, Knutsson \& Bøggild (6) show that shift work probably also increases the risk for peptic ulcer disease and gastrointestinal symptoms. Although a large amount of research has been carried out on the areas of sleep and sleepiness, cardiovascular health, cancer, and gastrointestinal diseases, even new avenues of research on shift work and health are emerging from the data, indicating that shift work may also increase the risk for type II diabetes and rheumatoid arthritis in addition to having a clear, although unspecific association with mental health (19-21).

Taking all the evidence together, shift work is probably related to a wide range of public health problems ranging from cardiovascular disease and cancer to mental health and accidents. Although the risk estimates for the outcomes are low (OR about $\leq 2$ ), the extensive exposure of workers (about $20 \%$ ) combined with the high prevalence of the diseases means that shift work is definitely among one of the most serious occupational health problems of our time.

Most of the published reviews on shift work and health underline the need to improve the quality of the published studies. Problems relating to exposure assessment, study design, and statistical analysis, as well as controlling for relevant confounders are still common, making the possibility for accurate 
interpretation of the studies' findings difficult. In the area of the shift work and cardiovascular disease, it has perhaps been easiest to follow the development of the scientific quality of the research thanks to consecutive reviews that have been conducted every ten years $(9,22,23)$. Although follow-up studies with large samples have become common, making the controlling of several confounders possible, the exposure assessment of many studies is still inferior. Costa et al (4) underline the need for better methods of proper exposure assessment in their discussion paper on the exposure assessment methodology of existing breast cancer studies.

Long gone is the time when shift workers were employed in the same factories for years, in the same regular shift systems. Shift work research faces new challenges as the "world of work" evolves with everincreasing speed, resulting in continuous changes to working hours and work demands. Workers also face changes in employment and may shift from one occupation to another. Researchers should focus on individual monitoring of shift work exposure rather than only expanding the study samples without improving the exposure assessment simultaneously. In many cases, the larger the sample, the weaker the exposure assessment; several of the largest studies have only estimated the average proportion of "shift work-exposed" individuals (4). In order to have accurate data on long-term exposure, exposure assessment should be done continuously throughout the follow-up period and include also information on the specific shift characteristics of the shift systems employed (eg, fixed/permanent shifts, amount of night shifts, and starting times, speed, and direction of shift rotation).

The next step may be the incorporation of "shift work biomarkers" into epidemiologic studies. As far back as the 1970s, biomarkers have been used in exposure monitoring and their application has since expanded from metals and organic solvents to many other risk factors of exposure (24). Melatonin is suppressed by light-at-night and could basically serve as a biomarker for exposure to night work. Light exposure, melatonin, and sleep deprivation are all also linked to the genetic regulation of human circadian rhythms and sleep $(4,25)$. It is possible that some genetic variants or changes among night shift workers could be used as future biomarkers for shift work or indications of increased sensitivity to work that involves occasional sleep deprivation and circadian adaptation.

Knowledge on the pathways and mechanisms mediating the effects of shift work to health is indeed necessary for new ideas in exposure assessment, analysis and interpretation of the epidemiological data. Moreover, the selection of the most appropriate prevention method is strongly influenced by whether the health effects of shift work are due to sleep deprivation, circadian disturbance, or psychosocial problems related to unsocial working hours. Shift work can probably disturb health by way of several psychosocial, behavioral, and physiological mechanisms. The multilevel endocrinological and immunological changes due to circadian disturbance, sleep loss, and melatonin suppression resulting from light-at-night may lead to the increased risk for endocrine tumors (4). For cardiovascular disease, the pathways probably include behavioral and psychosocial factors in addition to physiological mechanisms (3). With respect to gastrointestinal diseases, changing eating habits at work may be important (5) in addition to the circadian rhythm-related changes in gastrointestinal functions.

While epidemiology can tell us whether or not shift work is a health risk, it cannot really explain how shifts should be organized. In their review, Sallinen \& Kecklund (8) suggest that sleep and workrelated alertness are ameliorated by reducing the number of consecutive night shifts, avoiding long or early-morning shifts, and permitting sufficient time for sleep and rest between work shifts. However, the beneficial effects are rather modest and, even with a sleep-friendly shift system that minimizes circadian disruption, sleepiness is still relatively high during nighttime. This observation highlights the need for individual-based countermeasures in addition to the introduction of good shift systems. Pallesen et al (7) show that naps, bright-light exposure, and the use of stimulants such as coffee have proven to be effective alertness-enhancing countermeasures in laboratory-simulated night work. There are relatively few well-controlled trials in real shift work settings, but the results of the field studies are promising. 
Sallinen \& Kecklund (8) discuss that the individual's possibility to influence his or her work hours may mediate the relationship between the design of the shift system and its effects on sleep, alertness, and possibly also health. In Scandinavian countries, it is common in certain sectors (eg, healthcare) to have shift systems in which the individual can select his or her own work hours in line with the employer's staffing needs on various days and shifts. However, very little is known about how such shift systems affect sleep, alertness, and health. On the one hand, several prospective studies show that high control of employee work time is associated with good health and low levels of sickness absence and early retirement (26). On the other hand, there is also a risk that certain groups of shift workers may prioritize social needs and frequently select long work hours and short rest breaks between shifts, which in turn may increase the risk for adverse health consequences. Shift workers' knowledge of how they should organize work hours in order to avoid poor sleep and high levels of fatigue is, therefore, probably a key factor for the health consequences of individual-based flexible shift systems.

This special theme issue of the Scandinavian Journal of Work, Environment \& Health confirms that we have sufficient knowledge to conclude that shift work may indeed have detrimental consequences for health, despite the lack of information on all the possible health outcomes and the knowledge gaps related to exposure assessment and specific disease mechanisms. There is also a fairly large body of evidence showing how the negative consequences of shift work can be prevented by countermeasures on both the individual and organizational level, although well-controlled intervention studies are rare. Considering the pervasiveness of shift work and its numerous and serious health effects, we cannot continue to delay taking preventive and curative public health actions in anticipation of further data. In the workplace, more systematic and precise risk assessment for shift work is needed, as well as the prevention, detection, and treatment of shift work-related problems. Although there is a need for more specific information on the numerous health effects and pathways, we simultaneously need solution- and intervention-oriented research to alleviate the health problems of shift work today.

\section{References}

1. Costa G, Akerstedt T, Nachreiner F, Baltieri F, Carvalhais J, Folkard S, et al. Flexible working hours, health, and well-being in Europe: some considerations from a SALTSA project. Chronobiol Int. 2004;21:831-44.

2. Parent-Thirion A, Fernández Macías E, Hurley J, Vermeylen G. Fourth European Working Conditions Survey. Dublin: European Foundation for the Improvement of Living and Working Conditions; 2007.

3. Puttonen S, Härmä M, Hublin C. Shift work and cardiovascular disease - pathways from circadian stress to morbidity [review]. Scand J Work Environ Health. 2010;36(2):96-108.

4. Costa G, Haus E, Stevens R. Shift work and cancer - considerations on rationale, mechanisms, and epidemiology [discussion paper]. Scand J Work Environ Health. 2010;36(2):163-79.

5. Lowden A, Moreno C, Holmbäck U, Lennernäs M, Tucker P. Eating and shift work - effects on habits, metabolism, and performance [discussion paper]. Scand J Work Environ Health. 2010;36(2):150-62.

6. Knutsson A, Bøggild H. Gastrointestinal disorders among shift workers [review]. Scand J Work Environ Health. 2010;36(2):85-95.

7. Pallesen S, Bjorvatn B, Magerøy N, Saksvik IB, Waage S, Moen BE, et al. Measures to counteract the negative effects of night work [review]. Scand J Work Environ Health. 2010;36(2):109-20.

8. Sallinen M, Kecklund G. Shift work, sleep, and sleepiness - differences between shift schedules and systems. Scand J Work Environ Health. 2010;36(2):121-33.

9. Frost P, Kolstad HA, Bonde JP. Shift work and the risk of ischemic heart disease - a systematic review of the epidemiologic evidence [review]. Scand J Work Environ Health. 2009;35(3):163-79.

10. Kolstad HA. Nightshift work and risk of breast cancer and other cancers - a critical review of the epidemiologic evidence [review]. Scand J Work Environ Health. 2008;34(1):5-22.

11. Härmä M. Shift work among women - a century-old health issue in occupational health [editorial]. Scand J Work Environ Health. 2008;34(1):1-3.

12. Härmä M. Workhours in relation to work stress, recovery and health [review]. Scand J Work Environ Health 2006;32(6, special issue):502-514.

13. Ursin R, Baste V, Moen BE. Sleep duration and sleep-related problems in different occupations in the Hordaland Health Study. Scand J Work Environ Health. 2009;35(3):193-202.

14. Viitasalo K, Kuosma E, Laitinen J, Härmä M. Effects of shift rotation and the flexibility of a shift system on daytime alertness and cardiovascular risk factors. Scand J Work Environ Health. 2008;34(3):198-205. 
15. Chung M-H, Kuo TBJ, Hsu N, Chu H, Chou K-R, Yang CCH. Sleep and autonomic nervous system changes - enhanced cardiac sympathetic modulations during sleep in permanent night shift nurses. Scand J Work Environ Health. 2009;35(3):180-7.

16. Kecklund G. Long workhours are a safety risk — causes and practical legislative implications [editorial]. Scand J Work Environ Health 2005;31(5):325-7.

17. Pesch B, Harth V, Rabstein S, Baisch C, Schiffermann M, Pallapies D, et al. Night work and breast cancer - results from the German GENICA study. Scand J Work Environ Health. 2010;36(2):134-41.

18. Suwazono Y, Uetani M, Oishi M, Tanaka K, Morimoto H, Nakada S, et al. Estimation of the benchmark duration of alternating shift work associated with increased total cholesterol level among male Japanese workers. Scand J Work Environ Health. 2010;36(2):142-9.

19. Kawachi I, Colditz GA, Stampfer MJ, Willett WC, Manson JE, Speizer FE, et al. Prospective study of shift work and risk of coronary heart disease in women. Circulation. 1995;92:3178-82.

20. Puttonen S, Oksanen T, Vahtera J, Pentti J, Virtanen M, Salo P, et al. Is shift work a risk factor for rheumatoid arthritis? The Finnish Public Sector Study. Ann Rheum Dis. In press.

21. Bara A, Arber S. Working shifts and mental health - findings from the British Household Panel Survey (1995-2005). Scand J Work Environ Health. 2009;35(5):361-7.

22. Kristensen TS. Cardiovascular diseases and the work environment: a critical review of the epidemiologic literature on nonchemical factors [review]. Scand J Work Environ Health. 1989;15:165-79.

23. Bøggild H, Knutsson A. Shift work, risk factors, and cardiovascular disease. Scand J Work Environ Health. 1999;25:85-99.

24. Vainio H. Genetic biomarkers and occupational epidemiology - recollections, reflections and reconsiderations [editorial]. Scand J Work Environ Health. 2004;30(1):1-3.

25. Franken P, Dijk K. Circadian clock genes and sleep homeostasis. Eur J Neurosci. 2009;29(9):1820-9.

26. Vahtera J, Laine S, Virtanen M, Oksanen T, Koskinen A, Pentti J, et al. Employee control over working times and the risk of cause-specific disability pension: the Finnish Public Sector Study. Occup Environ Med. In press.

Mikko Härmä, MD, PhD, Finnish Institute of Occupational Health Topeliuksenkatu 41a A Fl 00250 Helsinki, Finland [E-mail: mikko.harma@ttl.fi]
Göran Kecklund, PhD

Stress Research Institute, Stockholm University, SE 10691 Stockholm, Sweden [E-mail: goran.kecklund@stressforskning.su.se] 